\title{
Changes in the activity of adenosine deaminase and the content of polyamines in the blood of cats with breast cancer
}

\author{
Maya Vakulenko ${ }^{1, *}$, Natalya Akinina ${ }^{2}$, Natalya Dobaeva ${ }^{2}$,Elena Bakurova ${ }^{3}$, Alexey \\ Ermakov ${ }^{4}$ \\ ${ }^{1}$ Southern Federal University, pr/ Stachki 194/1, Rostov-on-Don, Russia \\ ${ }^{2}$ Rostov State Medical University, Nakhichevansky Lane 29, Rostov-on-Don, Russia \\ ${ }^{3}$ National Medical University of Maxim Gorky, Illyica Ave 16, Donetsk, Ukraine \\ ${ }^{4}$ Don State Technical University, sq. Gagarina, 1, Rostov-on-Don, Russia
}

\begin{abstract}
In this work, the clinical significance of changes in the activity of adenosine deaminase (ADA) and the content of polyamines in the blood plasma of cats with breast cancer was studied. There were examined 30 cats aged 1 to 20 years, operated on in veterinary clinics due to spontaneous neoplasms. Histological studies of surgical material were performed to establish nosological forms of the tumor. The results of the measurements showed that there were no significant differences between ADA activity in the blood plasma of cats diagnosed with invasive nonspecific carcinoma and ADA activity in the blood of healthy animals. In the plasma of animals diagnosed with fibroadenomatous breast hyperplasia, ADA activity was reduced by $35 \%$ compared with the control group. The content of putrescine in the red blood cells of cats with benign neoplasms of the mammary gland (fibroepithelial hyperplasia) was statistically significantly increased 5 times compared with the control group. In malignant neoplasms of the mammary gland (invasive nonspecific carcinoma, tubular cancer and fibrosarcoma), the content of putrescine and spermine in the blood significantly exceeded the control values by 60 and 10 times, respectively, compared with the control group. Thus, the data obtained indicate the possibility of using polyamines in order to improve the accuracy of diagnosis and treatment of cats with breast cancer. The determination of ADA activity in the blood plasma of cats with breast tumors in the early stages of cancer is not an informationally significant indicator as a tumor biomarker.
\end{abstract}

\section{Introduction}

Breast neoplasm is one of the most common oncopathology among small domestic animals. Breast cancer in dogs is in the second place in frequency of occurrence, in cats in third place and differs from other forms of cancer by its aggressive course, malignant nature and poor prognosis $[1,2,3]$. The foregoing makes researchers look for new biomarkers of

* Corresponding author: vera.murgul@mail.ru 
breast cancer and develop cost-effective clinical analysis methods to monitor treatment efficacy $[4,5,6]$. One of the main features of cancerous tumors is the uncontrolled growth of degenerated cells. Active cell proliferation is accompanied by a change in purine metabolism. Derivatives of purines and their metabolites are involved in the synthesis of protein, DNA, RNA, and in many other biochemical reactions. For example, purine derivatives are necessary for the maturation and proliferation of immunocompetent cells, a violation of the purine mechanism affects the processes of immunoregulation and a decrease in immunity $[7,8,9,10,11]$. With the above, it can be assumed that carcinogenesis will be associated with a change in enzyme activity catalyzing the reaction of purine metabolism. Violation of the metabolism of polyamines is considered as one of the molecular mechanisms underlying the pathogenesis of cancer. Polyamines are compounds containing many amino groups. They have a low molecular weight, and are polyfunctional compounds. Polyamines include: spermidine, spermine and diamines - putrescine and cadaverine. In most mammals, and in particular in felines, normally polyamines are present in the blood in extremely low concentrations. The red blood cells take on the function of transporting polyamines; accordingly, in whole blood their level of content will depend on the level of their concentration in the formed elements. Serum and plasma spermine levels are lower than spermidine and putrescine. The amount of spermidine in whole blood predominates. The study of polyamine metabolism in the invivo and invitro experiments, as well as clinical and biochemical examinations of sick people with breast cancer, showed that the tumor development process is often accompanied by the accumulation of polyamines in the body. Therefore, their concentration in red blood cells and in blood as a whole is generally considered an indicator of cell proliferation. This indicator has clinical significance mainly when monitoring the dynamics of treatment of cancer patients. Activation of spermidine / spermine acetyltransferase (SSAT) in the tumor tissue causes the formation of several acetyl polyamine derivatives in high concentrations: $1 \mathrm{~N}$-acetyl spermidine, $8 \mathrm{~N}$-acetyl spermidine, $12 \mathrm{~N}$-acetyl spermidine, acetyl spermine, etc. The formation of acetyl derivatives of polyamines is associated with reduction of positive charge of molecule and leads to a weakening of their relationship with binding sites with DNA and RNA, which is pathogenetically associated with the progression of the pathological process. Currently, the determination of the content of acetyl derivatives of polyamines in saliva, blood plasma and urine is supposed to be used as a marker of oncology of the intestines, lungs, etc. Cells of tumor tissues usually have a high level of proliferation and, therefore, the synthesis of polyamines is increased. The level of increase in the concentration of polyamines in red blood cells in cancer patients has a direct relationship with the stage of the disease. In turn, this gives the right to consider the quantitative indicator of the polyamine content in erythrocytes as tumor markers, and use the change in this indicator to assess the effectiveness of the therapy [12]. Given the fact that domestic animals live next to humans and are subject to the same carcinogenic factors as humans, the study of tumor markers in domestic animals is an important component for the development of cancer science.

\section{Purpose of the study}

The purpose of this study was to learn the clinical significance and to establish the possibility of using as a tumor marker for breast cancer in cats, the determination of adenosine deaminase activity and the content of polyamines in the peripheral blood of sick animals.

\section{Materials and methods}


The object of the study was tissue of breast tumors and blood plasma of cats with neoplasms. The blood of healthy animals was used as a control; they were admitted to the hospital for sterilization and were selected on the basis of pairs of analogues. Histological studies were carried out on the basis of the educational laboratory of the Department of Biology and General Pathology, DSTU, the city of Rostov-on-Don. Biochemical studies were carried out on the basis of the biochemical laboratory of the Department of Biochemistry at Rostov State Medical University in Rostov-on-Don. Animals were selected for research among patients who came to the clinic for spontaneous tumors in the area of mammary glands. All cats with tumor lesions of the breast were prescribed surgical treatment. At the same time, the patient's age, the degree of operational risk, the size of the neoplasm and the absence of metastases were taken into account (according to the ultrasound and x-ray examination of the chest and abdominal cavity of the body). First of all, the treatment consisted of a uni- and bilateral resection of the mammary glands affected by tumor and regional lymph nodes. The study group included animals with 1 and 2 cancer stage, classification according to TNM (Owen, 1980). All animals had negative results for viral leukemia in cats FeLV and viral immunodeficiency in cats FIV. The operation was performed in compliance with the principles of ablastic and antiblastic. As anesthesia, standard algorithms for introducing into general anesthesia using intramuscular injection of Zoletil were used. The research protocol was in accordance with the guidelines of the Ethics Committee for Work with Animals of the Federal State Budget Educational Institution of Higher Education of the Rostov State Medical University on July 26, 2018. Operational material was photographed, measured, described and fixed in $10 \%$ buffered formalin for 24 hours. After fixation, histological preparations (paraffin blocks and glasses) were cut and manufactured according to the standard procedure [1]. The diagnosis of invasive non-specific breast carcinoma and hyperplastic changes was made according to the nomenclature and criteria of histological classification of breast tumors of the World Health Organization 2012, indicating the degree of differentiation [12].

The studied animals were divided into groups on the basis of tumor malignancy and degree of differentiation. The control group included healthy animals whose blood was taken during sterilization. A group of animals with benign neoplasms were animals with fibroepithelial hyperplasia. Groups of animals with malignant neoplasms were animals with invasive non-specific carcinoma of low, moderate and high degree of differentiation.

Enzyme activity was determined according to the Kalear method (Kalear, 1944) on a NanoProtometrImplen spectrophotometer. This method is based on a change in the optical density of the investigated fluid at a wavelength of $260 \mathrm{~nm}$. The change in density is due to the deamination of adenosine to inosine. In optical samples, a $0.36 \times 10-4 \mathrm{M}$ solution of adenosine in phosphate buffer $(\mathrm{pH}=7)$ was used as a substrate. As the enzymatic fraction used diluted blood plasma in a buffer in a ratio of 1: $270(0.01 \mathrm{ml}$ of blood plasma in $2.7 \mathrm{ml}$ of buffer). Reactions were carried out at a solution temperature of $5{ }^{\circ} \mathrm{C}$. ADA activity was calculated by the formula $\mathrm{A}=\Delta \mathrm{D} \times 1000 / \mathrm{t} \times 0.0149 \times 267 \mathrm{nmol} / \mathrm{s} / \mathrm{ml}$ plasma. Where $\Delta \mathrm{D}$ is the difference between the readings after and before incubation, 1000 is the dilution, $t$ is the time in seconds, 0.0149 is the coefficient from the ratio of the spectrophotometer reading when measuring the standard adenosine solution, 267 is the molecular weight of adenosine. The unit of enzyme activity was taken as such a quantity that ensured the conversion of $1 \mathrm{nmol}$ of adenosine in 1 second into $1 \mathrm{ml}$ of plasma under appropriate reaction conditions.

The polyamine content was determined in venous blood erythrocytes by high performance liquid chromatography on a Lichrospher RP18 column, previously converting them to benzoyl derivatives for identification in UV at $229 \mathrm{~nm}$. To obtain red blood cells, the blood was centrifuged for $10 \mathrm{~min}$ at $3000 \mathrm{rpm}$. The red blood cells washed with physiological saline were stored at $-30{ }^{\circ} \mathrm{C}$. Polyamines were determined in red blood cells 
obtained from $1 \mathrm{ml}$ of blood. To precipitate proteins, $1 \mathrm{ml}$ of $10 \%$ trichloroacetic acid was added to the samples, which was then neutralized with $2 \mathrm{ml}$ of $2 \mathrm{M} \mathrm{NaOH}$. Next, the samples were mixed with $20 \mu \mathrm{l}$ of benzoyl chloride (diluted in methanol 1:1). After vigorously stirring the mixture of samples with benzoyl chloride for 2 hours, $1 \mathrm{ml}$ of chloroform was added and centrifuged for $10 \mathrm{~min}$ at $350 \mathrm{~g}$. The chloroform fraction containing polyamines was collected, chloroform was re-added to the samples and centrifuged. As an internal control, 1,4-diaminoheptane (DAG) was used. Chloroform was evaporated at $80{ }^{\circ} \mathrm{C}$. The dry residue was dissolved in $100 \mu \mathrm{l}$ of $60 \%$ methanol. The composition of the mobile phase was methanol: water (60:40), the volume of the introduced sample was $20 \mu \mathrm{l}$, the chromatographic analysis time was $60 \mathrm{~min}$. The release time of benzoyl derivatives was 17 minutes for putrescine, 29-30 minutes for spermidine, 33 minutes for DAG, 49 minutes for spermine.

Statistical data processing was performed using the program "Statistica 6.0". The following statistical criteria were used to assess the reliability of the detected changes: Wilcoxon test (comparison of parameters measured in the control group and animals with breast tumors) and Student's test (comparison of two independent data groups). Differences between groups were considered significant at $p \leq 0.05$. Results are expressed as mean and mean error.

\section{Results of study and its discussion}

\section{Implen NanoPhotometer ${ }^{\circledR}$}

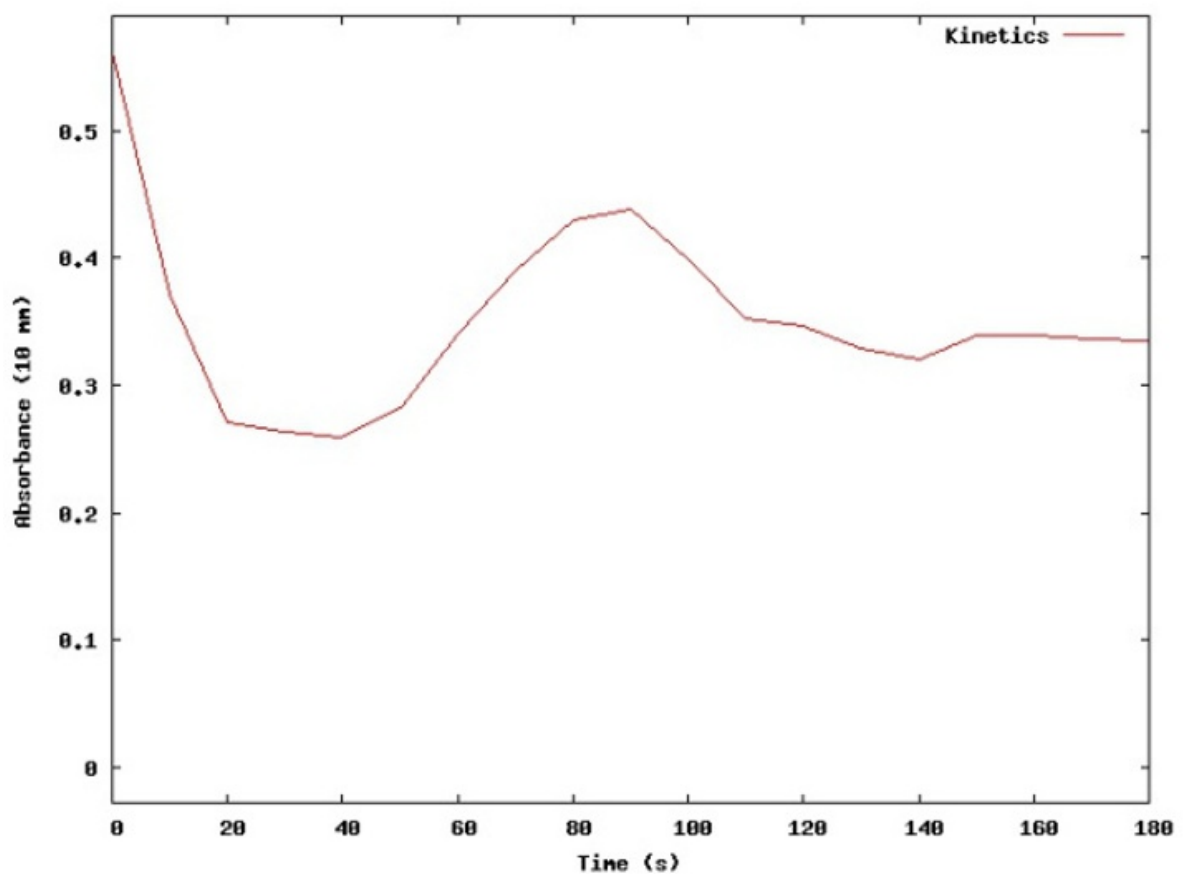

Fig. 1. Measurement of feline plasma adenosine deaminase activity.

The results of the study of ADA activity are presented in table 1.

Table 1. ADA activity in the blood plasma of healthy cats and cats with neoplasms ( $\mathrm{M} \pm \mathrm{m}$, in $\mathrm{nmol} /$ $\mathrm{sec} / \mathrm{ml}$ of blood plasma). 


\begin{tabular}{|c|c|}
\hline Groups & ADA Enzyme Activity \\
\hline Control & $67 \pm 5$ \\
\hline $\begin{array}{l}\text { All neoplasms } \\
\mathrm{n}=30\end{array}$ & $54 \pm 5(\mathrm{t}=1.00, \mathrm{p}=0.212)$ \\
\hline $\begin{array}{l}\text { Fibroepithelial hyperplasia } \\
\qquad \mathrm{n}=4\end{array}$ & $\begin{array}{c}45 \pm 2(\mathrm{t}=5.00, \mathrm{p}= \\
0.007)^{*}\end{array}$ \\
\hline $\begin{array}{l}\text { Highly differentiated invasive non-specific carcinoma } \\
\qquad \mathrm{n}=10\end{array}$ & $\begin{array}{l}50 \pm 10(\mathrm{t}=1.271, \mathrm{p}= \\
0.261)\end{array}$ \\
\hline $\begin{array}{l}\text { Moderately differentiated invasive non-specific carcinoma } \\
\qquad \mathrm{n}=9\end{array}$ & $47 \pm 2(t=1.33, p=0.22)$ \\
\hline $\begin{array}{l}\text { Low differentiated invasive non-specific carcinoma } \\
\qquad \mathrm{n}=7\end{array}$ & $\begin{array}{c}68 \pm 12(\mathrm{t}=0.278, \mathrm{p}= \\
0.79)\end{array}$ \\
\hline
\end{tabular}

The table shows that there were no significant differences between the ADA activity in the blood plasma of healthy animals and in the blood plasma of animals with malignant neoplasms of the mammary gland. In the blood plasma of animals with benign neoplasms, the ADA activity was reduced by $35 \%(\mathrm{p} \leq 0.05)$ relative to the control. No significant differences in enzyme activity depending on the degree of tumor differentiation were observed.

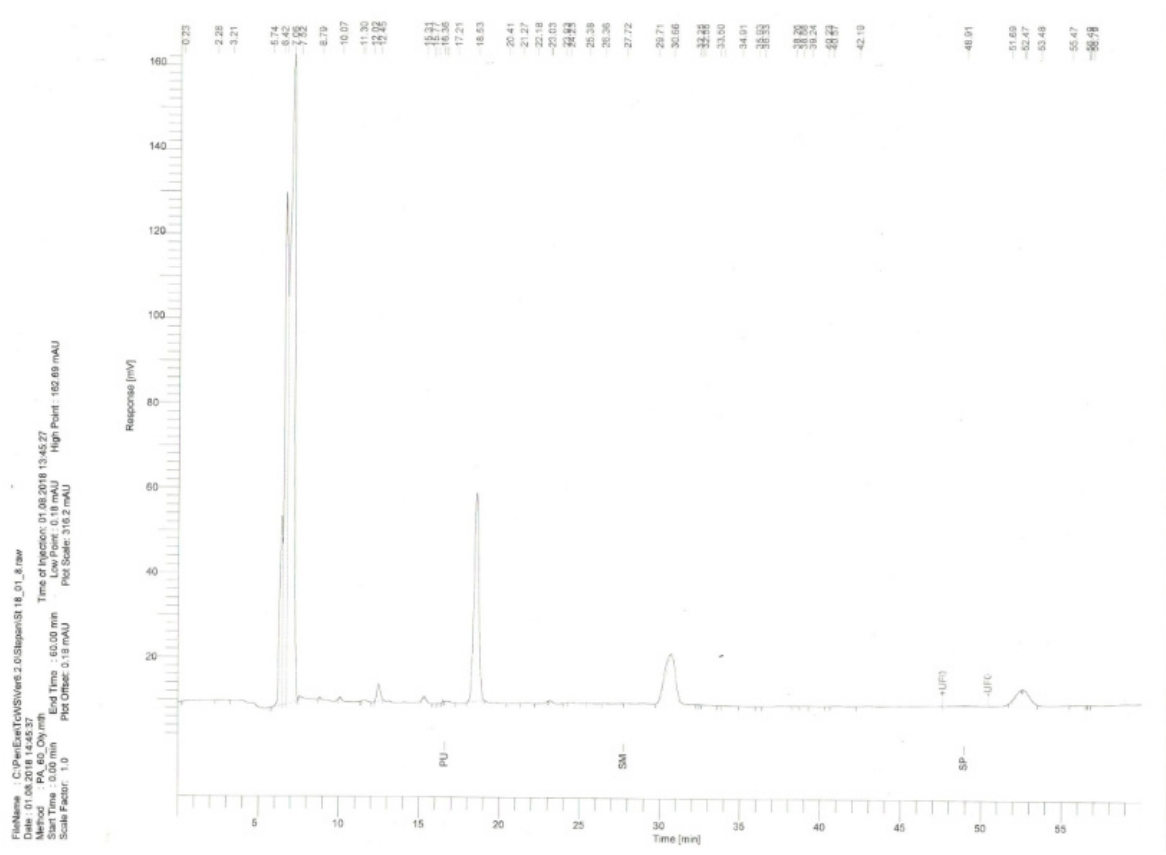

Fig. 2. Measurement of the content of polyamines and their derivatives in the peripheral blood of cats.

The content of polyamines in the blood erythrocytes of healthy cats is: putrescine - 0.04 $\mu \mathrm{g} / \mathrm{ml}$, spermidine $-5.94 \mu \mathrm{g} / \mathrm{ml}$ and spermine $-1.63 \mu \mathrm{g} / \mathrm{ml}$. These results of determining the content of polyamines in red blood cells correspond to the data published in the literature. The formation of benign neoplasms does not significantly affect the content of polyamines. The content of putrescine in the blood erythrocytes of cats with benign neoplasms of the mammary gland (fibroepithelial hyperplasia) statistically significantly increased 5 times $(p<0.0001)$ compared with the control group. Spermine content increased by $65 \%$, but this increase was not statistically significant. In case of malignant neoplasms of the mammary gland (invasive non-specific carcinoma), the content of putrescine and spermine in the 
blood significantly exceeded the control values by $60(p<0.0001)$ and 10 times $(p<0.02)$, respectively. At the same time, the content of spermidine in the blood of cats, on the contrary, decreased by 8 times $(\mathrm{p}<0.02)$ compared with the control (Table 2 ).

Table 2. The content of polyamines in the blood erythrocytes of cats with benign and malignant neoplasms of the mammary gland. ( $\mathrm{M} \pm \mathrm{m}$, in $\mu \mathrm{g} / \mathrm{ml}$ of blood, $\mathrm{n}=7-15)$.

\begin{tabular}{|c|c|c|c|}
\hline \multicolumn{4}{|c|}{ Polyamines, $\mu \mathrm{g} / \mathrm{ml}$} \\
\hline Groups & Putrescin & Spermidine & Spermine \\
\hline Clinical norm & $0.04 \pm 0.01$ & $5.94 \pm 1.13$ & $1.63 \pm 0.30$ \\
\hline Benign neoplasms & $2.15 \pm 0.22^{* *}$ & $4.00 \pm 0.40$ & $2.68 \pm 1.00$ \\
\hline Malignant neoplasms & $2.45 \pm 0.67^{*}$ & $0.76 \pm 0.14^{*}$ & $15.56 \pm 3.76^{*}$ \\
\hline
\end{tabular}

* - p1<0.02 - significance of differences in values in patients compared with the norm.

** - p $1<0.0001$ - significance of differences in values in patients compared with the norm.

We also noticed that in the tumor tissue (invasive non-specific carcinoma), the content of $1 \mathrm{~N}$-acetyl spermidine, $12 \mathrm{~N}$-acetyl spermidine and acetyl spermine is $30(\mathrm{p} \leq 0.001), 35$ $(\mathrm{p} \leq 0.001)$ and $10(\mathrm{p} \leq 0.01)$ times higher than in control.

At the same time, with this form of malignant neoplasms of the mammary gland in red blood cells, the presence of a high concentration of only one derivative, $12 \mathrm{~N}$-acetyl spermidine, was found to be 50 times greater than the control $(\mathrm{p} \leq 0.001)$.

\section{Conclusion}

- The use of ADA activity measurement according to the Kalear method as a tumor marker of breast cancer in cats is not advisable.

- The content of putrescine in the blood erythrocytes of cats with benign neoplasms of the mammary gland (fibroepithelial hyperplasia) statistically significantly increased 5 times in comparison with the control group. The content of spermine and spermidine did not change.

- In case of malignant neoplasms of the mammary gland (invasive non-specific carcinoma), the content of putrescine and spermine in the blood significantly exceeded the control values by 60 and 10 times, respectively, compared with the control. At the same time, the content of spermidine in the blood of cats, on the contrary, decreased by 8 times compared with the control.

- Obtained data indicate the possibility of using polyamines (spermidine, spermine, diamines) and their derivatives (acetyl spermidine) in order to improve the accuracy of diagnosis and treatment of breast cancer in cats.

\section{References}

1. B. Overley-Adamson, J. Baez, Feline internal medicine, 7, pp. 578-584 (2016) doi.org/10.1016/B978-0-323-22652-3.00059-1

2. O. Garden, S. Volk, N. Masson, J. Perry, The Veterinary Journal, 240, pp. 6-13 (2018) doi.org/10.1016/j.tvj1.2018.08.008

3. J. Withrow, D. Vail, L. Rodney, Withrow, MacEwen's, Small Animal Clinical Oncology, 5, pp. 1-29 (2013) doi.org/10.1016/B978-1-4377-2362-5.00001-3

4. A. Matos, A. Santos, 2015 The Veterinary Journal, 205, pp. 136-143 (2015) doi.org/10.1016/j.tvj1.2015.02.004

5. K. Hughes, J. Dobson, The Veterinary Journal, 194, pp. 19-26 (2012) doi.org/10.1016/j.tvj1.2012.05.008 
6. A. Matosab, C. Baptistaac, M. Gärtnerad, G. Ruttemane, The Veterinary Journal, 193, pp. 24-31 (2013) doi.org/10.1016/j.tvj1.2011.12.019

7. K. Abbed, H. El-Said Hamdia, Y.EI. Askar Basma, A. Kady, The Egyptian Rheumatologist, 05, 005 (2018) doi.org/10.1016/j.ejr.2018.05.005

8. V. Kim, A. Pham-Huy, E. Grunebaum, Journal of Allergy and Clinical Immunology, 143, pp. 403-405 (2019) doi.org/10.1016/j.jaci.2018.04.029

9. L. Castilhos, S. Adefegha, P. Doleski, T. Bertoldo, C. Eduardo, J. Moritz, E. Casal, Leal, Journal of Applied Biomedicine, 16, pp. 208-213 (2018) doi.org/10.1016/j.jab.2017.12.004

10. Z. Namiot, A. Kemona, J. Stasiewicz, M. Marcinkiewicz, A. Namiot, J. Gorsk, Cancer Letters, 82, pp, 95-98 (1994) doi.org/10.1016/0304-3835(94)90151-1

11. V. Battist, L. Maders, M. Bagatini, E. Battisti, L. Bellé, K. Santos, P. Maldonado, G. Thomé, M. Schetinger, M. Morsch, Biomedicine \& Pharmacotherapy, 67, pp. 203208 (2013) doi.org/10.1016/j.biopha.2012.12.004

12. G. Marverti, A. Ligabue, D. Guerrieri, G. Paglietti, S. Maria, D. Farina, Gynecologic Oncology, 117, pp. 202-210 (2010) doi.org/10.1016/j.ygyno.2009.11.030

13. A. Marx, J. Chan, J. Coindre, F. Detterbeck, Journal of Thoracic Oncology, 10, pp. 1383-1395 (2015) doi.org/10.1097/JTO.0000000000000654 